\title{
On the Statistics of Uplink Inter-Cell Interference with Greedy Resource Allocation
}

\author{
Hina Tabassum ${ }^{\# 1}$, Ferkan Yilmaz ${ }^{\# 2}$, Zaher Dawy ${ }^{* 3}$, and Mohamed Slim Alouini ${ }^{\# 4}$ \\ \# Physical Sciences and Engineering Division, \\ King Abdullah University of Science and Technology (KAUST), \\ Thuwal, Saudi Arabia \\ ${ }^{1,2,4}$ \{hina.tabassum, ferkan.yilmaz, slim.alouini\}@kaust.edu.sa \\ * Department of Electrical and Computer Engineering, \\ American University of Beirut (AUB), \\ Beirut, Lebanon \\ ${ }^{3}$ zd03@aub.edu. 1b
}

\begin{abstract}
In this paper, we introduce a new methodology to model the uplink inter-cell interference (ICI) in wireless cellular networks. The model takes into account both the effect of channel statistics (i.e., path loss, shadowing, fading) and the resource allocation scheme in the interfering cells. Firstly, we derive a semi-analytical expression for the distribution of the locations of the allocated user in a given cell considering greedy resource allocation with maximum signal-to-noise ratio (SNR) criterion. Based on this, we derive the distribution of the uplink ICI from one neighboring cell. Next, we compute the moment generating function (MGF) of the cumulative ICI observed from all neighboring cells and discuss some examples. Finally, we utilize the derived expressions to evaluate the outage probability in the network. In order to validate the accuracy of the developed semi-analytical expressions, we present comparison results with Monte Carlo simulations. The major benefit of the proposed mechanism is that it helps in estimating the distribution of ICI without the knowledge of instantaneous resource allocations in the neighbor cells. The proposed methodology applies to any shadowing and fading distributions. Moreover, it can be used to evaluate important network performance metrics numerically without the need for time-consuming Monte Carlo simulations.
\end{abstract}

Index Terms-multi-cell, inter-cell interference, greedy resource allocation.

\section{INTRODUCTION}

Modeling inter-cell interference (ICI) in cellular networks is a cornerstone step for devising efficient resource allocation schemes, developing interference mitigation algorithms, and evaluating system performance. The primary challenge comes from the fact that ICI depends on a wide range of system parameters that can be arbitrary such as the allocation of resources to users in neighboring cells and the frequency reuse pattern in the network.

In the next generation OFDMA-based cellular networks (e.g., WiMAX and LTE), the system bandwidth is decomposed into flat-faded orthogonal frequency bands denoted as subcarriers. These subcarriers are then allocated to the users within a cell according to some resource allocation scheme. Considering this orthogonality among the users in a cell, the intra-cell interference can be ignored. However with frequency reuse equal to one, the users allocated on the same subcarrier in the neighbor cells can cause severe ICI, depending on their distance from the base station (BS) of interest. Clearly, in such scenarios the primary cause of ICI on a given subcarrier are the single users allocated to that subcarrier in each neighbor cell. Thus, interference cannot be averaged out on each subcarrier. Moreover, it may not be accurate to model ICI as a Gaussian random variable (RV) by invoking the central limit theorem.
Interference modeling and analysis have been addressed in the literature using different approaches and scenarios. In [1], the authors derived analytical subcarrier collision models that capture the probability of allocating a subcarrier simultaneously in neighboring cells based on traffic load. They derived upper and lower bounds for the expected number of subcarrier collisions and analyzed the throughput performance in a multicell scenario. In [2], the signal-to-interferencenoise ratio (SINR) distribution is derived in semi-analytical form for the downlink of femtocell networks. The ICI is modeled considering the effect of path loss and shadowing. In [3], the authors investigated the effect of ICI in downlink multiuser diversity networks and presented a methodology to analyze system capacity. In [4], the authors investigated the applicability of the Gaussian and binomial distributions for downlink ICI modeling. They found that the Gaussian distribution approximates ICI more closely than the binomial distribution. They have also shown that the significant interference contribution is from two dominant interfering cells only irrespective of the user locations. Clearly, the result is valid only for downlink scenarios as the location of all interferers is fixed and deterministic.

In contrast to downlink, modeling of ICI in the uplink is more challenging due to the arbitrary locations of the interferers. The number of significantly contributing interferers in the uplink can vary based on their location which is highly dependent on the deployed resource allocation schemes. In [5], the authors developed an analytical model for collisions as a function of the cell load and frequency reuse schemes. Also, they derived an expression for the SINR in the uplink and downlink, ignoring the effect of shadowing and fading. Another interesting work is [6], in which the authors model ICI as a function of reuse partitioning radius and traffic load in OFDMA uplink systems. The resource allocation criterion is assumed to be random. In [7], the authors presented a semi-analytical method to analyze the ICI in uplink cellular networks. They approximate the distribution of uplink ICI through numerical simulations without considering the impact of resource allocation schemes.

We propose a new methodology to model uplink ICI based on both the channel statistics and the resource allocation scheme in the cellular networks. In this paper, we focus on modeling the ICI on a given subcarrier assuming greedy user selection, i.e., selecting the users based on maximum signal to noise ratio (SNR) criterion. The resource allocation scheme is assumed to be identical in all the cells. We then utilize 
the derived ICI distribution to evaluate the outage probability in the network. The accuracy of the derived semi-analytical expressions is verified through Monte Carlo simulations.

The paper is organized as follows: Section II defines the system model and presents the proposed methodology. In Section III, we derive the distribution of the locations of the allocated users in a given cell. Based on this, in Section IV, we determine the distribution of the uplink ICI from a neighbor cell. In Section $\mathrm{V}$, we compute the moment generating function (MGF) of the cumulative ICI and present some examples. In Section VI, we investigate the outage probability of the network. Section VII presents the numerical results followed by concluding remarks in Section VIII.

Notation: Throughout the paper, $\operatorname{Exp}(\lambda)$ denotes an exponential distribution with parameter $\lambda, \operatorname{Gamma}\left(m_{s}, m_{c}\right)$ represents a Gamma distribution with shape parameter $m_{s}$ and scale parameter $m_{c} . \Gamma($.$) represents the Gamma function. p(A)$ denotes the probability of event A. $\{a, b\}$ denotes a discrete set of elements which ranges from $a$ to $b$. Finally, $\mathbb{E}[$.$] denotes$ the expectation operator.

\section{System Model And Proposed Methodology}

We consider a given cell surrounded by $L$ neighbor cells. The cells are assumed to be circular with same number of users. The user distribution is considered to be uniform in all the cells. However, the methodology can be extended to any number of users and any arbitrary user distribution. Each cell $l$ has radius $R$ with a total of $U$ users. The average transmit power of each user in the uplink is assumed to be unity. We assume all users transmit with maximum power with rate adaptation depending on their channel qualities. Thus, each user has an instantaneous SNR $\gamma$ based on its location, shadowing, and fading statistics yielding:

$$
\gamma=r^{-\beta} S F=r^{-\beta} \zeta
$$

where $r$ is the user distance from its own BS. $\beta$ is the path loss exponent, $S$ and $F$ represent the shadowing and fading components, respectively, and $\zeta$ represents a combined shadowing and fading component.

Depending on the radius and user density in each cell, we decompose the cellular region into $K$ concentric circular rings. The circular regions between the two adjacent rings are characterized by uniform path loss variation (in $\mathrm{dB}$ ) and thus, possesses non-uniform width $\Delta_{k}$. Since, path loss varies exponentially, $\Delta_{k}$ continues to increase from cell center to cell edge. The average number of users in a given ring $k$ can be computed as follows:

$$
u_{k}=\frac{U\left(r_{k}^{2}-r_{k-1}^{2}\right)}{R^{2}} \quad k=1,2, \ldots, K
$$

where $r_{k}$ denotes the radius of ring $k$. The basic motivation behind discretizing the cellular region is complexity reduction since users within each circular ring have similar channel conditions, i.e., approximately the same path loss as $\Delta_{k} \rightarrow 0$ and $K \rightarrow \infty$. The proposed methodology to model uplink ICI is based on the following steps:

1) Derive the distribution $f_{r_{\mathrm{sel}}}(r)$ of allocating the given subcarrier to a user at a distance $r_{\text {sel }}$ from its serving BS based on a maximum SNR criterion. Since we assume identical resource allocation scheme implemented in all cells, $f_{r_{\text {sel }}}(r)$ remains same for all cells.

2) Derive the distribution of the distance between the allocated users in the neighbor interfering cells and the BS of the cell of interest, i.e., determine $f_{\tilde{r}_{\text {sel }}}(\tilde{r})$ using $f_{r_{\mathrm{sel}}}(r)$ where $\tilde{r}_{\text {sel }}$ is the distance of the allocated interfering users from the BS of interest.

3 ) Derive the distribution of the interference $X_{l}$ from the allocated user in neighboring cell $l$ to the BS of interest. $X_{l}$ can be written as:

$$
X_{l}=\tilde{r}_{\text {sel }}^{-\beta} \chi
$$

where, $\chi$ denotes the combined shadowing and fading component of the interference statistics. $f_{X_{l}}(x)$ can be determined by averaging the composite fading distribution $f_{\chi}(\chi)$ over $f_{\tilde{r}_{\text {sel }}}(\tilde{r})$.

4) Derive the MGF of the cumulative interference caused by the allocated users in all interfering cells. The cumulative interference can be written as $Y=\sum_{l=1}^{L} X_{l}$.

\section{Distribution of the Allocated User Locations}

Considering the intricacy of deriving a continuous probability density function (PDF), here we discuss a simple approach to determine the discrete distribution of the distance of selected user in a given cell, i.e., the probability mass function (PMF) of $r_{\text {sel }}$ which consists of the following two steps:

Step 1 (Selecting the user with the highest SNR in ring $k$ ): Since each circular region has uniform path loss variation, the users within a ring $k$ are assumed to be subject to approximately the same path loss. Thus, selecting a user in a ring $k$ is equivalent to selecting the user with maximum channel gain among all the users in ring $k$ i.e.,

$$
\zeta_{k}=\max \left(\zeta_{1}, \zeta_{2}, \ldots, \zeta_{u_{k}}\right)
$$

Note that, here $\zeta_{1}, \zeta_{2}, \cdots \zeta_{u_{k}}$ are independent and identically distributed (i.i.d) channel gains of the users based on shadowing and fading component. Thus, for any ring $k$, the cumulative distribution function (CDF) and PDF of the maximum channel gain $\zeta_{k}$ can be written as:

$$
\begin{gathered}
F_{\zeta_{k}}\left(\zeta_{k}\right)=\left(F_{\zeta}\left(\zeta_{k}\right)\right)^{u_{k}} \\
f_{\zeta_{k}}\left(\zeta_{k}\right)=u_{k} f_{\zeta}\left(\zeta_{k}\right)\left(F_{\zeta}\left(\zeta_{k}\right)\right)^{u_{k}-1}
\end{gathered}
$$

In order to take the path loss into account, we can now perform a simple transformation of RVs. From (1), $\gamma_{k}=r_{k}^{-\beta} \zeta_{k}$, where, $\gamma_{k}$ is the selected user SNR in each ring $k$. Thus, the CDF and PDF of $\gamma_{k}$ can be written as follows:

$$
\begin{gathered}
F_{\gamma_{k}}\left(\gamma_{k}\right)=\left(F_{\zeta}\left(\gamma_{k} r_{k}^{\beta}\right)\right)^{u_{k}} \\
f_{\gamma_{k}}\left(\gamma_{k}\right)=\frac{u_{k}}{r_{k}^{-\beta}} f_{\zeta}\left(\gamma_{k} r_{k}^{\beta}\right)\left(F_{\zeta}\left(\gamma_{k} r_{k}^{\beta}\right)\right)^{u_{k}-1}
\end{gathered}
$$

Step 2 (Selecting the best ring $k$ from the $K$ rings): In this step, we need to compute the probability of selecting $k^{\text {th }}$ ring among all other rings. It is important to note that this is equivalent to selecting the ring $k$ which possesses the user with the highest SNR among all rings. Thus, conditioning on $\gamma_{k}$, the PDF of $r_{\text {sel }}$ can be written explicitly as follows:

$$
P\left(r_{\mathrm{sel}}=r_{k} \mid \gamma_{k}\right)=\prod_{i=1, i \neq k}^{K} p\left(\gamma_{i} \leq \gamma_{k}\right)=\prod_{i=1, i \neq k}^{K} F_{\gamma_{i}}\left(\gamma_{k}\right)
$$

By averaging over the distribution of $\gamma_{k}$, the final expression for the PMF of $r_{\text {sel }}$ is:

$$
P\left(r_{\mathrm{sel}}=r_{k}\right)=\int_{0}^{\infty}\left(\prod_{i=1, i \neq k}^{K} F_{\gamma_{i}}\left(\gamma_{k}\right)\right) f_{\gamma_{k}}\left(\gamma_{k}\right) d \gamma_{k}
$$


Using (7), (10) can be written as follows:

$$
P\left(r_{\text {sel }}=r_{k}\right)=\int_{0}^{\infty} \prod_{i=1, i \neq k}^{K}\left(F_{\zeta}\left(\gamma_{k} r_{i}^{\beta}\right)\right)^{u_{i}} f_{\gamma_{k}}\left(\gamma_{k}\right) d \gamma_{k}
$$

where $r_{\text {sel }} \in\{0, R\}$. Using (8), (11) can be written as:

$$
\int_{0}^{\infty} \prod_{i \neq k}^{K}\left(F_{\zeta}\left(\gamma_{k} r_{i}^{\beta}\right)\right)^{u_{i}} \frac{u_{k} f_{\zeta}\left(\gamma_{k} r_{k}^{\beta}\right)}{r_{k}^{-\beta}}\left(F_{\zeta}\left(\gamma_{k} r_{k}^{\beta}\right)\right)^{u_{k}-1} d \gamma_{k}
$$

The results in (12) are generalized for any shadowing and fading statistics. Even though (12) is not a closed form expression, the integration can be solved easily using standard software packages such as MAPLE and MATHEMATICA. Note that, $P\left(r_{\mathrm{sel}}=r_{k}\right)$ in (11) and (12) is the marginal PMF of $P\left(r_{\mathrm{sel}}=r_{k}, \theta=\theta_{i}\right)$ where $\theta$ is distributed uniformly from 0 to $2 \pi$. Although the PDF of $\theta$ is continuous we can discretize it in order to reduce complexity.

Consider discretizing the range of RV $\theta$ in $I$ uniform angular intervals of desired accuracy. Thus $P\left(\theta=\theta_{i}\right)=\frac{1}{I}$ where $\theta_{i}$ denotes any discrete value that the RV $\theta$ can take. Since $r_{\text {sel }}$ and $\theta$ are independent, their joint PMF can be written as follows:

$P\left(r_{\text {sel }}=r_{k}, \theta=\theta_{i}\right)=P\left(r_{\text {sel }}=r_{k}\right) P\left(\theta=\theta_{i}\right)=\frac{P\left(r_{\text {sel }}=r_{k}\right)}{I}$

\section{Distribution OF THE ICI}

In order to derive $f_{X_{l}}(x)$, firstly we need to find the distribution of the distance of users allocated in the interfering cell $l$ to the BS of interest, i.e., $f_{\tilde{r}_{s e l} l}(\tilde{r})$. Since, each cell is assumed to have identical conditions, $f_{\tilde{r}_{\mathrm{sel}}}(\tilde{r})$ remains the same for all interfering cells and we will not use subscript $l$ any further to avoid complexity of notations. Using the cosine law, we can write:

$$
\tilde{r}_{\mathrm{sel}}^{2}=r_{\mathrm{sel}}^{2}+D^{2}-2 r_{\mathrm{sel}} D \cos \theta
$$

where $\tilde{r}_{\text {sel }}$ is the distance of the selected interfering user in cell $l$ from the BS of interest, $r_{\mathrm{sel}}$ is the distance of the selected interfering user from its own BS, i.e., (BS $l$ ), $\theta \in\{0,2 \pi\}$ and $D$ is the reuse distance. Since, we consider universal frequency reuse and first tier of interfering cells, $D=2 R$. The method, however, can be extended to any number of tiers of interfering cells in a straightforward manner.

In order to determine the PMF of $\tilde{r}_{\text {sel }}$ where $\tilde{r}_{\text {sel }} \in\{D-$ $R, D+R\}$, first of all we compute $\tilde{r}_{i, k}$ for given $\theta_{i}$ and $r_{k}$ using (14) as follows:

$$
\tilde{r}_{i, k}^{2}=r_{k}^{2}+D^{2}-2 r_{k} D \cos \theta_{i} \quad \forall r_{k}, \forall \theta_{i}
$$

Clearly $\tilde{r}_{i, k}$ are the points at which $P\left(\tilde{r}_{\text {sel }}=\tilde{r}_{i, k}\right)$ can be defined using (13) as follows:

$$
P\left(\tilde{r}_{\mathrm{sel}}=\tilde{r}_{i, k}\right)=\frac{P\left(r_{\mathrm{sel}}=r_{k}\right)}{I}
$$

The two dimensional (2-D) data set of $\tilde{r}_{\text {sel }}$, at which $P\left(\tilde{r}_{\text {sel }}=\right.$ $\left.\tilde{r}_{i, k}\right)$ is defined, can then be grouped into $M$ arcs of any arbitrary width $\Delta$. This can be done by dividing the distance between $D-R$ and $D+R$ into $M$ equal arcs of width $\Delta$ and mapping $\tilde{r}_{i, k}$ accordingly. Clearly, by adding all the probabilities for which $\tilde{r}_{\text {sel }}$ lies in the $m^{\text {th }}$ arc we get the

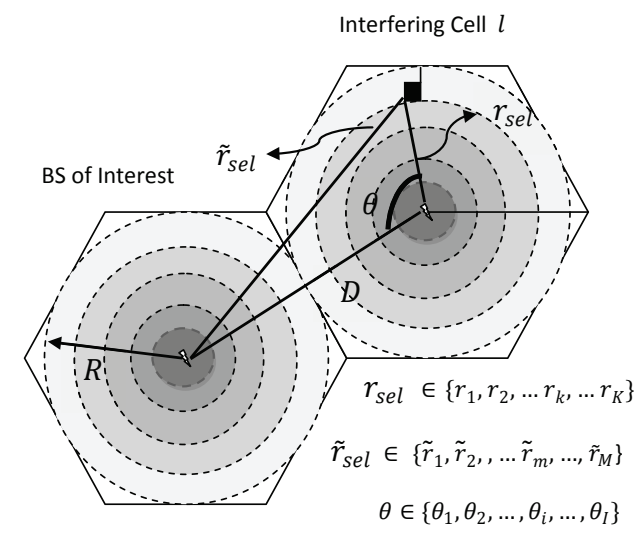

Fig. 1. Cellular network model with each cell divided into multiple rings.

probability of $\tilde{r}_{\mathrm{sel}}=\tilde{r}_{m}$ :

$$
P\left(\tilde{r}_{\text {sel }}=\tilde{r}_{m}\right)=\sum_{\tilde{r}_{i, k} \in\left\{\tilde{r}_{m}-\frac{\Delta}{2}, \tilde{r}_{m}+\frac{\Delta}{2}\right\}} P\left(\tilde{r}_{\text {sel }}=\tilde{r}_{i, k}\right)
$$

where $\tilde{r}_{m}$ denotes any discrete value that the RV $\tilde{r}_{\text {sel }}$ can take (See Fig. 1). Now, using (3) the PDF of $X$ conditioned on $\tilde{r}_{\text {sel }}$ can be determined by RV transformation as follows:

$$
f_{X \mid \tilde{r}_{\mathrm{sel}}}=\frac{f_{\chi}\left(x \tilde{r}_{\mathrm{sel}}^{\beta}\right)}{\tilde{r}_{\mathrm{sel}}^{-\beta}}
$$

Simply averaging over the PMF of $\tilde{r}_{\text {sel }}$ we can compute the distribution of interference, i.e., $f_{X}(x)$ by any cell $l$ as follows:.

$$
f_{X}(x)=\sum_{\tilde{r}_{m}=\tilde{r}_{1}}^{\tilde{r}_{M}} \frac{f_{\chi}\left(x \tilde{r}_{m}^{\beta}\right)}{\tilde{r}_{m}^{-\beta}} P\left(\tilde{r}_{\mathrm{sel}}=\tilde{r}_{m}\right)
$$

\section{MGF of the Cumulative ICI}

Computing the distribution of $Y$ is not a trivial task as it requires the convolution of RVs $X_{l}$ for $l=1,2, \cdots L$. However, assuming that all interferers are i.i.d., we can compute the MGF $\mathcal{M}_{Y}(t)$ of $Y$ simply as follows:

$$
\mathcal{M}_{Y}(t)=\prod_{l=1}^{L} \mathcal{M}_{X_{l}}(t)=\left(\mathcal{M}_{X}(t)\right)^{L}=\left(\mathbb{E}\left[e^{t x}\right]\right)^{L}
$$

Looking at the structure of (19), we can write $\mathcal{M}_{X}(t)$ as:

$$
\begin{gathered}
\int_{0}^{\infty} e^{t x} f_{X}(x) d x=\int_{0}^{\infty} e^{t x} \sum_{\tilde{r}_{m}=\tilde{r}_{1}}^{\tilde{r}_{M}} \frac{f_{\chi}\left(x \tilde{r}_{m}^{\beta}\right)}{\tilde{r}_{m}^{-\beta}} P\left(\tilde{r}_{\mathrm{sel}}=\tilde{r}_{m}\right) d x \\
\mathcal{M}_{X}(t)=\sum_{\tilde{r}_{m}=\tilde{r}_{1}}^{\tilde{r}_{M}} \frac{P\left(\tilde{r}_{\mathrm{sel}}=\tilde{r}_{m}\right)}{\tilde{r}_{m}^{-\beta}} \int_{0}^{\infty} e^{t x} f_{\chi}\left(x \tilde{r}_{m}^{\beta}\right) d x
\end{gathered}
$$

The derived expressions apply to any shadowing and fading distributions. Next, we will discuss the statistics of $X_{l}$ and $\mathcal{M}_{Y}(t)$ for two typical example scenarios.

Example 1 (Rayleigh Fading - $\zeta, \chi \sim \operatorname{Exp}(\lambda)$ ): Considering the simplest case of just Rayleigh fading (i.e., no shadowing) $f_{X}(x)$ can be written using (19) as follows:

$$
f_{X}(x)=\sum_{\tilde{r}_{m}=\tilde{r}_{1}}^{\tilde{r}_{M}} \lambda \tilde{r}_{m}^{\beta} e^{-\lambda \tilde{r}_{m}^{\beta} x} P\left(\tilde{r}_{\mathrm{sel}}=\tilde{r}_{m}\right)
$$


Note that (23) is a hyperexponential distribution with parameter $\lambda \tilde{r}_{m}^{\beta}$. Thus, using MGF of the hyperexponential distribution, $\mathcal{M}_{Y}(t)$ can be derived as follows:

$$
\mathcal{M}_{Y}(t)=\left(\sum_{\tilde{r}_{m}=\tilde{r}_{1}}^{\tilde{r}_{M}} \frac{\lambda \tilde{r}_{m}^{\beta}}{\lambda \tilde{r}_{m}^{\beta}-t} P\left(\tilde{r}_{\mathrm{sel}}=\tilde{r}_{m}\right)\right)^{L}
$$

Example 2 (Composite Fading - $\zeta, \chi \sim \operatorname{Gamma}\left(m_{s}, m_{c}\right)$ ): In this example, another important scenario is considered in which shadowing and fading statistics are modeled by a Gamma and Nakagami distribution, respectively. In this case, the composite distribution has a closed form which is known as generalized-K or Gamma-Gamma distribution [8]. However, the generalized-K distribution can be approximated by a simple Gamma distribution using moment matching method [9]. In this case, $f_{X}(x)$ can be written as follows:

$$
f_{X}(x)=\sum_{\tilde{r}_{m}=\tilde{r}_{1}}^{\tilde{r}_{M}} \frac{e^{-\frac{x \tilde{r}_{m}^{\beta}}{m_{c}}}\left(x \tilde{r}_{m}^{\beta}\right)^{m_{s}-1}}{\tilde{r}_{m}^{-\beta} \Gamma\left(m_{s}\right) m_{c}^{m_{s}}} P\left(\tilde{r}_{\mathrm{sel}}=\tilde{r}_{m}\right)
$$

Using (22), the expression for $\mathcal{M}_{Y}(t)$ can be derived as follows:

$$
\mathcal{M}_{Y}(t)=\left(\sum_{\tilde{r}_{m}=\tilde{r}_{1}}^{\tilde{r}_{M}} P\left(\tilde{r}_{\mathrm{sel}}=\tilde{r}_{m}\right)\left(\frac{\tilde{r}_{m}^{\beta}}{\tilde{r}_{m}^{\beta}-m_{c} t}\right)^{m_{s}}\right)^{L}
$$

Due to the limited space, the derivations are skipped here.

\section{Vi. Outage Probability Evaluation}

The derived ICI distribution expressions can be used to evaluate numerically important network performance metrics such as outage probability, ergodic capacity, and link budget interference margin. In this section, we demonstrate how the derived expressions can be used to compute the outage probability $P_{\text {out }}$ in the network. The evaluation of ergodic capacity is skipped, in this paper, due to space constraints.

To compute $P_{\text {out }}$, we follow the strategy introduced in [10]. Firstly, we define a new RV, $Z=q \sum_{l=1}^{L} X_{l}-X_{0}$, where $q$ is the outage threshold and $X_{0}$ is the corresponding signal power of the user of interest in the central cell. The outage event occur when $p(Z \geq 0)$. The characteristic function of $Z$ is $\phi_{Z}(\omega)=\mathbb{E}\left[e^{j Z \omega}\right]$. The general expression for $\phi_{Z}(\omega)$ can be written as follows:

$$
\phi_{Z}(\omega)=\phi_{Y}(q \omega) \phi_{X_{0}}(-\omega)
$$

Finally, we can compute $P_{\text {out }}$ by evaluating the definite integral [10] as follows:

$$
P_{\text {out }}=\frac{1}{2}+\frac{1}{2 \pi} \int_{-\infty}^{\infty} \operatorname{Im}\left(\frac{\phi_{Z}(\omega)}{\omega}\right) d \omega
$$

where $\operatorname{Im}\left(\phi_{Z}(\omega)\right)$ denotes the imaginary part of $\phi_{Z}(\omega)$. The expression for $\phi_{Z}(\omega)$ is presented for the first and second example scenario, respectively as follows:

$$
\begin{aligned}
\phi_{Z}(\omega)=\left(\sum_{\tilde{r}_{m}=\tilde{r}_{1}}^{\tilde{r}_{M}} \frac{\lambda \tilde{r}_{m}^{\beta}}{\lambda \tilde{r}_{m}^{\beta}-j q \omega} P\left(\tilde{r}_{\mathrm{sel}}=\tilde{r}_{m}\right)\right)^{L} \\
\quad \times \sum_{r_{k}=r_{1}}^{r_{K}} \frac{\lambda r_{k}^{\beta}}{\lambda r_{k}^{\beta}+j \omega} P\left(r_{\mathrm{sel}}=r_{k}\right)
\end{aligned}
$$

$$
\begin{aligned}
\phi_{Z}(\omega)= & \left(\sum_{\tilde{r}_{m}=\tilde{r}_{1}}^{\tilde{r}_{M}}\left(\frac{\tilde{r}_{m}^{\beta}}{\tilde{r}_{m}^{\beta}-j q \omega m_{c}}\right)^{m_{s}} P\left(\tilde{r}_{s e l}=\tilde{r}_{m}\right)\right)^{L} \\
& \times \sum_{r_{k}=r_{1}}^{r_{K}}\left(\frac{r_{k}^{\beta}}{r_{k}^{\beta}+j \omega m_{c}}\right)^{m_{s}} P\left(r_{\text {sel }}=r_{k}\right)
\end{aligned}
$$

\section{RESUlts AND ANALYSIS}

In this section, we will verify the accuracy of the derived semi-analytical expressions through Monte Carlo simulations. Due to the lack of space, the results are presented for the second example scenario, i.e., $f_{\zeta}(\zeta)$ is $\operatorname{Gamma}(0.5,2)$ and $f_{\chi}(\chi)$ is $\operatorname{Gamma}(3 / 2,2 / 3)$. However, the trend remains similar for the Rayleigh fading case. The radius $R$ of the cell is taken $500 \mathrm{~m}$ and the path loss exponent $\beta=2.6$. The cell is decomposed into non-uniform circular regions of width $\Delta_{k}$ each. The path loss variation within each region is set equal to $2 \mathrm{~dB}$. Firstly, we define the Monte Carlo simulation setup for obtaining the simulated results for all derived distributions.

1) Generate $U$ uniformly distributed users in a circular cell of radius $R$. Each user has SNR given by (1). Select the user with maximum SNR.

2) Store the distance of the selected user, i.e., $r_{\text {sel }}$, compute the distance of the selected user from the BS of interest i.e., $\tilde{r}_{\text {sel }}$ using cosine law and finally compute the interference $X$ using (3).

3) Repeat Step 1 and Step 2 for a large enough number of iterations (in this section, we run 100,000 iterations).

4) Generate histogram for discrete RV $r_{\text {sel }}$ with nonuniform bin widths and another histogram for discrete RV $\tilde{r}_{\text {sel }}$ with any arbitrary bin width equal to $\Delta$ (in this section, $\Delta=10 \mathrm{~m}$ ).

Fig. 2 depicts the PMF of the allocated users in any cell based on maximum SNR selection, for 100 and 200 users. It can be observed that as the number of competing users for a given subcarrier increases, the PMF gets skewed. This reflects the fact that the more the competing users, the greater is the chance of allocating a user near the cell center. The accuracy of the resulting PMF remains comparable to the exhaustive Monte Carlo simulations with only 25 rings. Since we consider uniform user distribution and narrow circular regions near the cell center, the concentration of users is extremely low in these regions. Thus, even though the chances of allocation are high near the cell center, the low user density causes the descend in the probability of allocation. Moreover, the path loss model in (1) is valid, in general, for distances greater than a threshold reference distance (typical ranges for $10-100 \mathrm{~m}$ outdoor and $1-10 \mathrm{~m}$ indoor). We select a reference distance of $10 \mathrm{~m}$ for the numerical evaluations and the simulations. Thus, for demonstration purpose we do not consider any user within $10 \mathrm{~m}$ which can be observed in Fig. 2.

In Fig. 3, the PMF of the distance at which users are allocated in the interfering cell $l$, i.e., $P\left(\tilde{r}_{\text {sel }}=\tilde{r}_{m}\right)$ is shown. Numerical results are found to be in agreement with the analytical results. The small descend can be clearly observed from $990-1010 \mathrm{~m}$ which is due to ignoring any user within the ring of radius $10 \mathrm{~m}$. Fig. 4 demonstrates the CDF and PDF of the continuous $\mathrm{RV} X$, i.e., ICI considering a single interfering cell. In Fig. 5, we evaluate the outage probability of the network for different number of users and path loss exponents. As $\beta$ increases the outage probability of the network is expected to be reduced for any number of users. Moreover, as the concentration of competing users increases for a given subcarrier, the probability of the selection of users possessing 

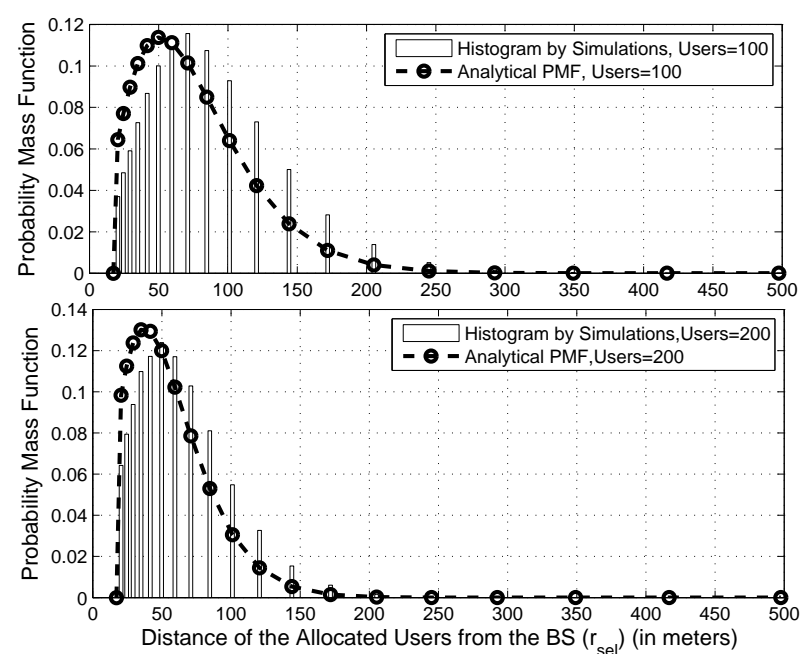

Fig. 2. PMF of the distance of allocated users in a cell (i.e., PMF of $r_{\text {sel }}$ )
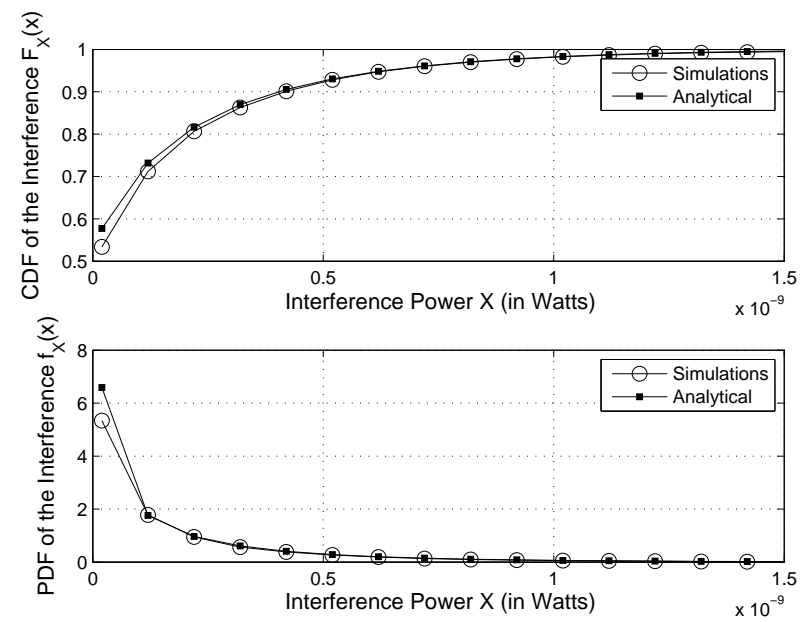

Fig. 4. CDF and PDF of the interference from cell $l$

good channel gains increases. This leads to the reduction of outage probability as the number of users increases.

\section{CONCLUSION}

In this paper, we have introduced a new methodology to model the uplink ICI considering both the channel statistics and the resource allocation scheme. The proposed mechanism helps in estimating the distribution of ICI without the knowledge of instantaneous resource allocations in the neighbor cells. The obtained results achieve the accuracy of Monte Carlo simulations with much reduced complexity. Furthermore, the methodology is generalized for various shadowing and fading distributions. The derived expressions can be used to evaluate important system performance metrics numerically such as outage probability and ergodic capacity.

\section{REFERENCES}

[1] S. Yarkan, K. Teo, H. Arslan, J. Zhang, J. and K.A. Qaraqe, "Upper and lower bounds on subcarrier collision for inter-cell interference scheduler in OFDMA based systems: Voice traffic," Physical Communication, vol. 3, no. 4, pp. 265-275, December, 2010.

[2] K.W. Sung, H. Haas, and S. McLaughlin, "A semianalytical PDF of downlink SINR for femtocell networks," EURASIP Journal on Wireless Communications and Networking, vol. January, 2010.

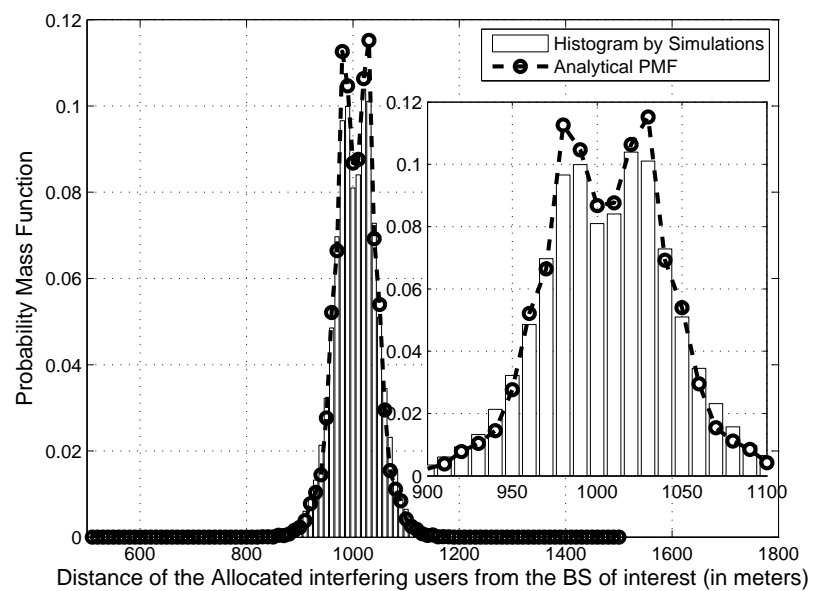

Fig. 3. PMF of the distance at which interfering users are allocated (i.e., PMF of $\left.\tilde{r}_{\text {sel }}\right), I=720$

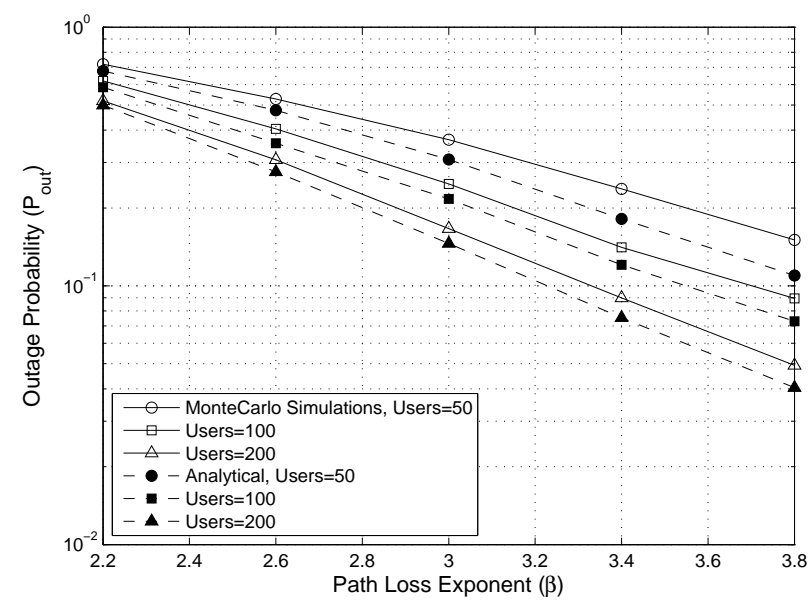

Fig. 5. Outage probability of the network $\left(P_{\text {out }}\right)$ against path loss exponent $(\beta)$ for various number of users, $q=18 \mathrm{~dB}$

[3] S.H. Kim, B.C. Jung, and D.K. Sung, "Effect of other-cell interference on multiuser diversity in cellular networks," International Symposium on Communications and Information Technologies. ISCIT'07, pp. 521-525, Sydney, Australia, October, 2007.

[4] S. Plass, X.G. Doukopoulos, and R. Legouable, "Investigations on linklevel inter-cell interference in OFDMA systems," IEEE Symposium on Communications and Vehicular Technology, pp. 49-52, Liege, November, 2006.

[5] S. Elayoubi, B. Haddada, and B. Fourestie, "Performance evaluation of frequency planning schemes in OFDMA based networks," IEEE Transactions on Wireless Communications, vol. 7, no. 5, pp. 1623-1633, May, 2008.

[6] E.Oh, M.G. Cho, S. Han, C. Woo, and D. Hong, "Performance analysis of reuse-partitioning-based subchannelized OFDMA uplink systems in multicell environments," IEEE Transactions on Vehicular Technology, vol. 57, no. 4, pp. 2617-2621, July, 2008.

[7] I.Viering, A. Klein, M. Ivrlac, M. Castaneda, and J.A. Nossek, "On uplink intercell interference in a cellular system," IEEE International Conference on Communications, ICC'06, vol. 5, pp. 2095-2100, Istanbul, Turkey, June, 2006.

[8] P. M. Shankar, "Error rates in generalized shadowed fading channels," Wireless Personal Communications, vol. 28, no. 3, pp. 233-238, 2004

[9] S. Al-Ahmadi and H. Yanikomeroglu, "On the approximation of the generalized-K PDF by a Gamma PDF using the moment matching method," Wireless Communications and Networking Conference, WCNC'09, pp. 1-6, Budapest, Hungary, April 2009.

[10] Q.T. Zhang, "Outage probability of cellular mobile radio in the presence of multiple Nakagami interferers with arbitrary fading parameters," IEEE Transactions on Vehicular Technology, vol. 44, no. 3, pp. 364-372, May, 1996. 